\title{
OTIOTOMICS
}

Revista de economía, empresa y sociedad

Dossier: «Repensando la enseñanza de la economía en la universidad»

ENTREVISTA

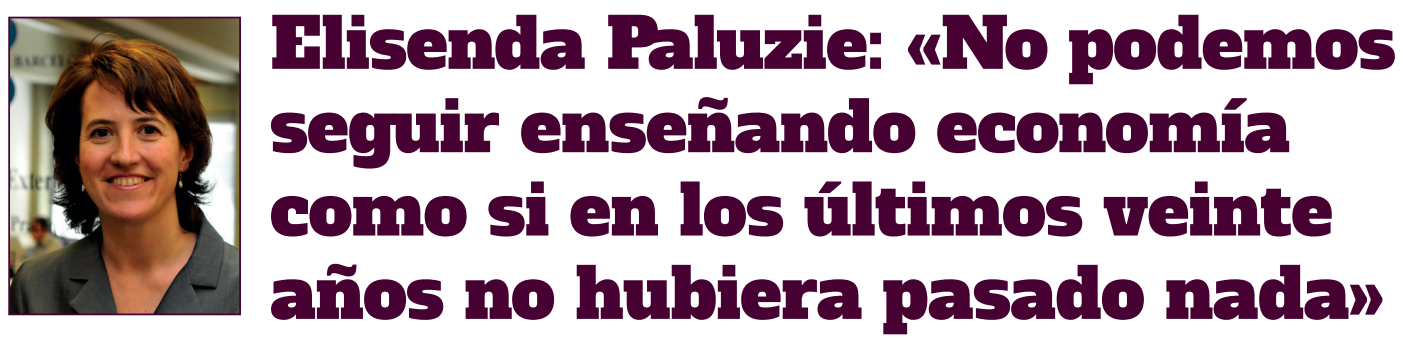

\section{Carolina Hintzmann}

Profesora de Estudios de Economía y Empresa (UOC)

\section{Joan Miquel Gomis}

Profesor de Estudios de Economía y Empresa (UOC)

RESUMEN En esta entrevista, Elisenda Paluzie analiza los orígenes del denominado movimiento Post-Crash en Cataluña y cuáles han sido los efectos sobre una institución referente como la que dirige: la Facultad de Economía y Empresa de la Universidad de Barcelona (UB). Paluzie considera que este movimiento crítico iniciado tras el inicio de la última crisis económica se fundamenta básicamente en la falta de pluralidad en la enseñanza a partir de tres elementos: el teórico, el metodológico y el interdisciplinario. En este texto comenta cómo son tratados estos tres factores en su facultad y concluye la necesidad de evolucionar en la docencia en la economía. En este sentido destaca el proyecto The core Project, una iniciativa que tiene por objeto replantear la enseñanza de la economía con nuevos manuales, ya que considera que los dominantes actualmente siguen planteando las cuestiones como si los últimos veinte años no hubieran existido.

PALABRAS CLAVE Post-Crash; enseñanza de la economía; pluralismo; teoría neoclásica; teoría económica; EEES

\section{Elisenda Paluzie: "We can't go on teaching economics as if nothing had happened over the last 20 years»}

SUMMARY In this interview Elisenda Paluzie analyses the origins of the so-called Post Crash movement in Catalonia and the effects on a renowned institution such as the one she heads: the Faculty of Economics and Business at the University of Barcelona (UB). Paluzie believes this critical movement, which began following the recent economic crisis, is principally rooted in a lack of plurality in teaching, which can be based on three elements: theory, methodology and interdisciplinarity. She comments on how these three factors are treated in her faculty and ends on the need for the teaching of economics to evolve. In this regard she highlights 'The core Project', an initiative with a goal to reset the approach to economics teaching with new coursebooks, in the belief that the materials currently holding sway continue to approach matters as if during the last 20 years nothing had occurred. 
KEYWORDS post crash; teaching of economics; pluralism; neoclassical theory; economic theory; EHEA

Elisenda Paluzie es la decana de la Facultad de Economía y Empresa de la Universidad de Barcelona (UB). Doctora en Economía por la UB, ha sido estudiante visitante de investigación en la London School of Economics y tiene un máster en Economía Internacional y Desarrollo por la Universidad de Yale. Como responsable de una facultad de Economía y Empresa referente en nuestro sistema universitario, con una larga historia y con un elevado número de estudiantes, sus opiniones sobre la orientación de los estudios de economía son relevantes.

Carolina Hintzmann / Joan Miquel Gomis: ¿Qué valoración hace de la corriente crítica sobre la docencia de la economía que podemos denominar Post-Crash?

Elisenda Paluzie: En el caso de Cataluña, el movimiento que denominamos Post-Crash se origina con estudiantes de la Universidad Pompeu Fabra, a los que se unen estudiantes de otras universidades, entre ellas la nuestra. Después de la última crisis económica, estos estudiantes inician un movimiento crítico que se fundamenta básicamente en lo que consideran una falta de pluralidad en la enseñanza de la economía desde tres puntos de vista: el teórico, el metodológico y el interdisciplinario.

C.H. / J.M.G.: Desde el punto de vista teórico, esta corriente crítica señala que en los planes de estudio solo se presenta una sola escuela de pensamiento. ¿También es así en su facultad?

E.P.: Este movimiento Post-Crash, efectivamente, denuncia que la escuela de pensamiento económico que predomina en los planes de estudio es la neoclásica, con variaciones internas pero excluyendo la economía heterodoxa, como la poskeynesiana, la institucional, la ecológica, la feminista, la marxista y la austríaca. En nuestra facultad seguimos el paradigma dominante y el núcleo duro de la enseñanza de la economía es la microeconomía y la macroeconomía con una vertiente dominante ortodoxa, si así lo queremos denominar.

Pero esto no quiere decir que en nuestro grado de Economía no tengamos algunas asignaturas optativas que reflejan visiones más heterodoxas, aunque tengan un papel menor. Es el caso de asignaturas como Economía ecológica o Economía feminista. También en la asignatura Economía mundial presentamos un enfoque diferente y heterodoxo al hablar, por ejemplo, de teorías de la dependencia que nacen de una raíz marxista.

C.H. / J.M.G.: ¿Y desde el punto de vista metodológico?

E.P.: La crítica a la falta de pluralidad metodológica se centra en aspectos como la evaluación, por ejemplo, con la excesiva utilización de pruebas tipos test o la práctica ausencia de ensayos para evaluar al estudiante, además de la carencia de pensamiento crítico, la desconexión entre la teoría y la realidad, y la potenciación de métodos cuantitativos en detrimento de los cualitativos.

En parte, esta crítica es pertinente para nuestro grado porque es cierto que podía existir una cierta desconexión entre realidad y teoría en una época anterior, cuando los grupos de estudiantes eran muy numerosos y se hacía docencia con manuales estadounidenses, dando lugar a que los estudiantes trabajaran ejemplos y problemas económicos no siempre ajustados a nuestra realidad. Pero con la implementación del Espacio Europeo de Enseñanza Superior (EEES), esto se está corrigiendo y cada vez hay más asignaturas en las que el profesorado hace un esfuerzo para, a través de los mecanismos de evaluación continuada, hacer trabajar a los estudiantes de manera más eficiente para relacionar la teoría y la realidad económica.

Nuestro Departamento de Teoría Económica es un buen ejemplo de ello, con iniciativas metodológicas innovadoras que pretenden mejorar la percepción que el estudiante tiene de la teoría y de la realidad. Es el caso del proyecto que ha introducido en las aulas el trabajo para analizar, tratar, comparar e interpretar datos trabajando por parejas de estudiantes en casos que ponen de manifiesto la relación entre teoría y realidad. En este contexto, 
se incluye un ejercicio en el que los estudiantes hacen una simulación práctica en un juego en el que asumen roles de jefes de estado y de gobernadores de bancos centrales. Los estudiantes, por grupos, según los roles asignados, han de tomar decisiones de política monetaria en un debate preparado para facilitar elementos de discusión sobre estas cuestiones.

Por otro lado, aunque la evaluación tipo test todavía existe, con el desarrollo del EEES ha aumentado la pluralidad de los métodos de evaluación, entre ellos los ensayos, que han ganado un cierto peso en algunas asignaturas y especialmente a través del trabajo final de grado, asignatura obligatoria para todos los estudiantes.

C.H. / J.M.G.: ¿Y en cuanto a la carencia de interdisciplinariedad que denuncia esta corriente crítica?

E.P.: Una de las grandes quejas que se expresan en cuanto a este aspecto es que prácticamente han desaparecido de los planes de estudios asignaturas como Historia económica, Historia del pensamiento económico, Sociología, Ciencia políica, Filosofía de la economía o Ética. Pero creo que por lo que respecta a esta cuestión nuestro plan de estudios, a diferencia de otras facultades, no puede ser objeto de crítica, puesto que incorpora dos asignaturas de Historia económica obligatorias y una de Historia del pensamiento económico también obligatoria. Además, tenemos como obligatoria la asignatura Sociología, y como optativas Ciencia política y Filosofía de la economía. Este es un factor distintivo que nuestra facultad ha mantenido, forma parte de nuestro ADN porque creemos que se ha de tener una perspectiva histórica de la evolución de la economía, tanto desde el punto de vista de la historia económica como de la historia del pensamiento, para situar las diferentes teorías.

C.H. / J.M.G.: ¿Cómo se puede avanzar más en estos tres ejes (teórico, metodológico, interdisciplinar) que estamos comentando?

E.P.: Hay un proyecto que a mí me parece muy interesante; creo que va por buen camino y considero que hay que seguirlo. Es el llamado The Core Project (http://www.core-econ.org/), que lideran profesores como Sam Bowles, del Instituto Santa Fe, o Wendy Carlin, de la University College de Londres, con un amplio grupo de profesores de varias universidades, entre las que figuran las clásicas anglosajonas. Una de las iniciativas que han llevado a cabo es la elaboración de un manual para enseñar análisis económico, que tiene por objetivo replantear la enseñanza de la economía porque consideran que la mayoría de manuales siguen enseñando economía como si los últimos veinte años no hubieran existido. Es un tema que va más allá del pluralismo ideológico, para centrarse en la evolución de la economía y en la inclusión de nuevos puntos de vista, como el de la economía del comportamiento, que comprende aspectos como el de la ética en diferentes contextos y facilita la colaboración entre psicólogos y economistas. Estos puntos de vista no se contemplan en los manuales tradicionales, pues siguen priorizando el análisis del comportamiento de los agentes.

Este manual introduce los adelantos que han tenido lugar en la economía y que han modificado o están modificando aspectos sustanciales de su evolución, además de intentar aproximar teoría y realidad. El planteamiento del manual, que mantiene aspectos convencionales, incorpora elementos nuevos, como las interacciones sociales, el medio ambiente, la desigualdad, la propiedad y el poder, o el análisis de la crisis actual, entre otros muchos. Este proyecto incide también en el análisis de las instituciones, puesto que identifica el contexto institucional de un país como un factor determinante para la actividad económica. Se trata de temas que no abundan en los manuales tradicionales, pero que en cambio sí se han desarrollado en la investigación de la economía.

En definitiva, me parece una iniciativa muy interesante que parte de la identificación de la necesidad de evolucionar en la docencia de la economía. Un proyecto que hay que seguir por las aportaciones que puede hacer en este debate.

C.H. / J.M.G.: El debate generado por esta corriente crítica se ha centrado mucho en los planes de estudio de economía, pero en las facultades de Economía y Empresa como la suya la mayor parte de los estudiantes están matriculados en las titulaciones de Administración y Dirección de Empresas (ADE). Parece que esta titulación no entra en el debate...

E.P.: Efectivamente, los estudiantes que firman el manifiesto son de titulaciones de Economía y, por tanto, sus reflexiones se han centrado en este ámbito de conocimiento. Pero en realidad, la reflexión tiene que ser general, porque tenemos que pensar en los perfiles de economista que nos da cada titulación. Con la distin- 
ción entre las titulaciones de ADE y Economía nos encontramos con el hecho de que claramente formamos a perfiles diferentes. Hay una tendencia a la hiperespecialización, y esto puede ser un problema porque nos podemos encontrar con que materias fundamentales pueden perder peso en una carrera o en la otra. Por ejemplo, podemos pensar que los estudiantes de ADE cursan pocas asignaturas de economía, cuando el análisis del entorno económico es básico para la gestión de las empresas. Y a la inversa, en los planes de estudio de Economía suele haber pocas asignaturas sobre contabilidad... y quizás estamos formando economistas que después no saben interpretar un balance. Al tener dos grados separados quizás estamos dispersando el conocimiento necesario.

C.H. / J.M.G.: Esta reflexión sobre el perfil del economista ¿cómo influye en el debate sobre la posible implementación de grados de ciento ochenta créditos?

E.P.: En el caso de revisiones de planes de estudio, por ejemplo, ante la posible implementación de grados de ciento ochenta créditos, y partiendo de la base de que, por la propia definición del EEES, los grados han de ser generalistas, yo entiendo de entrada que esta visión histórica que hemos comentado, y que nos tiene que enseñar a pensar de manera interdisciplinar, se ha de mantener. Un grado generalista también debe enseñar a pensar y a desarrollar la capacidad crítica.

La tendencia del sistema universitario español es a diseñar grados de ADE y de Economía diferenciados y creo que aunque se hagan grados de tres años, no se harán cambios en este sentido. Pero en otros países la realidad es diferente. En Francia, por ejemplo, el equivalente al grado de tres años es común entre empresa y economía, y es a partir de los másters cuando los estudiantes se especializan.

J.C.H. / J.M.G.: ¿Son necesarios doscientos cuarenta créditos en un grado de economía?

E.P.: Entiendo que doscientos cuarenta créditos son necesarios, pero también considero que los cuatro años que implican estos créditos se pueden fraccionar en tres más uno, de forma que se facilite al estudiante una titulación progresiva que le puede ayudar a avanzar en su carrera académica y a lograr una especialización con el máster después de una titulación generalista.

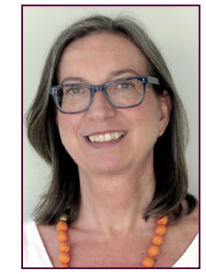

Garolina Hintzmann

chintzmann@uoc.edu

Profesora de los Estudios de Economía y Empresa (UOC)

Doctora en Economía por la Universidad de Barcelona y licenciada en Ciencias Económicas y Empresariales por la misma universidad. Directora del grado de Economía de los Estudios de Economía y Empresa de la UOC y coordinadora de movilidad internacional de los mencionados estudios. Sus áreas de especialización se sitúan en el ámbito de la macroeconomía, el crecimiento económico, la productividad del trabajo y el e-Learning. Es miembro del grupo de investigación Management \& e-Learning (MeL). 


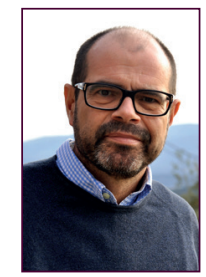

\section{Joan Miquel Gomis}

jgomis|@uoc.edu

Profesor de los Estudios de Economía y Empresa (UOC)

Director de Oikonomics. Doctor por el Departamento de Economía y Organización de Empresas de la Universidad de Barcelona. Licenciado en Ciencias de la Información por la UAB. Director del programa (grado) de Turismo de los Estudios de Economía y Empresa de la UOC. Ha sido codirector académico (2006-2010) del máster sobre Gestión, Política y Estrategia de los Destinos Turísticos, organizado conjuntamente por la UOC y la Organización Mundial del Turismo (OMT). Sus áreas de especialización se sitúan en el ámbito de la dirección estratégica de la empresa turística, la aplicación de las TIC a la distribución y en el ámbito de la responsabilidad social. Investigador del grupo consolidado GRATET y miembro del Laboratorio del Nuevo Turismo de la UOC.

Los textos publicados en esta revista están -si no se indica lo contrario- bajo una licencia Reconocimiento-Sin obras derivadas 3.0 España de Creative Commons. Puede copiarlos, distribuirlos y comunicarlos públicamente siempre que cite su autor y la revista y la institución que los publica (autoría, nombre de la revista, institución editora); no haga con ellos obras derivadas. La licencia completa se puede consultar en http://creativecommons.org/licenses/by-nd/3.0/es/deed.es.

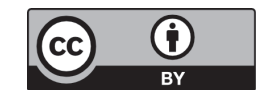

